Gazi University
Journal of Science
http://dergipark.gov.tr/gujs

\title{
The Current-Voltage Characteristics for Electrode Geometry Model of Positive DC Corona Discharge in Air
}

\author{
Asep Yoyo WARDAYA ${ }^{1,2^{*}}$ (D), Zaenul MUHLISIN ${ }^{1}$, Jatmiko Endro SUSENO ${ }^{(1 D)}$, Muhammad NUR ${ }^{1}$, \\ Pandji TRIADYAKSA ${ }^{(D)}$, Ali KHUMAENI ${ }^{10}$, Susilo HADI ${ }^{1}$, Eko Adi SARWOKO ${ }^{\text {(D) }}$, \\ Jaka WINDARTA ${ }^{2}$ \\ ${ }^{I}$ Department of Physics, Faculty of Science and Mathematics, Diponegoro University, Semarang Indonesia \\ ${ }^{2}$ Master Program of Energy, School of Post Graduate Studies, Diponegoro University, Semarang Indonesia \\ ${ }^{3}$ Department of Computer Science/Informatics, Faculty of Science and Mathematics, Diponegoro University, Semarang Indonesia
}

\author{
Highlights \\ - This paper focuses on the $(I-V)$ characteristics calculations of the corona discharge. \\ - The numerical calculation approach uses the concept of the electrode geometry model. \\ - There is an accuracy high degree between the numerical calculations and the research data.
}

Article Info
Received: 23 Feb 2021
Accepted: 08 Aug 2021
Keywords
Corona discharge
(I-V) characteristics curve
PSC-P
Python GUI Programming
k-shape sharpness factor

\section{INTRODUCTION}

At present, the use of electronic equipment with corona plasma discharge technology is increasingly being produced by industry and is in demand by the various public. The concept of plasma discharge as a form of ionized gas in an electric discharge [1] has been widely applied to the model of plasma reactor equipment referred to as capacitive coupled plasma (CCP) [2,3]. The CCP model is composed of two electrodes in a perpendicular to each other position, with electrode 1 in a stand up straight position with the bottom edge of the plate has a sharp form, while electrode 2 has a flat surface in a position lying below electrode 1 [4].

The corona plasma discharge can be generated by an ac or dc voltage source. The appearance of the plasma discharge is caused by various physical events in the plasma such as EHD flow [5,6], convective heat transfer [7], electric wind [8,9], electrostatic precipitation [10,11], etc. The corona discharge process begins with a flat graph (Townsend event) and continues with a sharp increase in a function's graph of the currents to voltage (corona event) [12]. Most of the corona discharge events will appear exit from the sharp surface of electrode 1 headed for electrode 2 because the sharp geometry surface will have a high potential gradient [4].

The research we conducted was to discuss the $(I-V)$ characteristic of positive dc corona discharge in air. The CCP electrode configuration used in this study is the Point and Semicircular-Plane (PSC-P). This 
configuration consisting of two electrodes perpendicular to each other, where the two electrodes are made of copper material. The first electrode in an upright position with a very thin plate thickness has the shape of a rectangular plate which is perforated in a semicircular in the middle and has a sharp shape on both sides of the bottom of the plate, whereas the second electrode has the form as a horizontal plate in a position lying under electrode 1.

Dobranszky et al. [13] have published research results about the plasma size (plasma height and width) of the corona discharge produced by variations in the electrode taper angle of $10^{\circ}, 20^{\circ}, 30^{\circ}, 45^{\circ}, 60^{\circ}$, and $90^{\circ}$. In addition to experiments on variations in angles, there are also 3 different material variations at the end of the electrode. The results of these experiments (for angles $30^{\circ}-90^{\circ}$ ) indicate that the size of the height and width of the plasma will increase if the electrode taper angle (in degree) becomes smaller for the three variations of the material.

Owning to this research that based on the experiment result of [13], it is created a comparability factor that was obtained from the results of the corona discharge experiment regarding the shape and size of the plasma which grew larger if the electrode tip grew tapered through a factor of the $k$-shape sharpness. The $k$ value also contributes greatly to the magnitude of the discharge of the plasma from the tapered electrode when compared to the shape of the electrode that is close to flat. The $k$-shape sharpness factor is manually multiplied by the capacitance value of the electrode which has only a sharp geometric shape. The $k$ value is obtained not through the concept of numerical calculation but through a comparison in fitting with experimental data.

\section{MATERIAL METHOD}

\subsection{Mathematical Models}

The Townsend relationship for the small distance from the point-plane electrode model has been expressed by Ferreira et al. [14] in the form as

$$
I=C_{1}\left(V-V_{i}\right)^{2},
$$

where $I$ is the corona current, $C_{1}$ is a constant, $V$ is the applied voltage and $V_{\mathrm{i}}$ is the corona threshold voltage. Furthermore, several other journals discuss the $(I-V)$ characteristics of the Townsend formula such as the fluid flow model of electrons and ions for the formulation of potential $V$ [15] and the EHD flow model [6]. There is also Townsend's low-current approach formula for Coaxial Cylinders in Air [16] which is expressed as

$$
I=10^{6} \frac{8 \pi \in b_{0}}{\delta R^{2} \ln (R / r)} V\left(V-V_{0}\right),
$$

where $V_{0}$ is the initial voltage of the corona, $\delta$ is the critical density of the gas (air), $R$ and $r$ respectively are the radius of the outer and inner electrodes and $b_{0}$ is ion mobility $[(\mathrm{m} / \mathrm{s}) /(\mathrm{V} / \mathrm{m})]$ at $\delta=1$.

In this study, there is a constant value of $C_{1}$ as expressed in formula (1) determined through the formulation of capacitance. In this formulation, we entering the corona discharge properties arising from the sharp electrode geometric shape of the PSC-P configuration model. Before calculating the capacitance of the PSC-P model, we must first calculate the simpler capacitance model, namely the line-plane capacitance model. The calculation of the PSC-P capacitance can be adopted from the line-plane model. An illustration of the PSC-P model in the case of the corona plasma discharges can be seen in the Figure 1(a). 


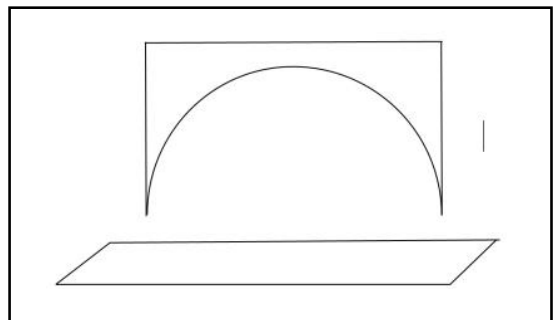

(a)

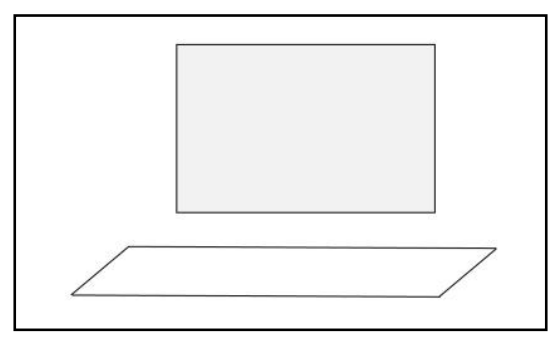

(b)

Figure 1. CCP reactor equipment for electrode configuration models of (a). PSC-P and (b) Line-Plane

To calculate the capacitance value of the PSC-P configuration model, we must adopt the capacitance value calculation model of the line-plane configuration model as shown in Figure 1 (b). The two configuration models, basically consisting of two plates in a perpendicular position each other, with a very thin plate standing upright on the other plate which is in a horizontal position. For the capacitance calculation of the Line-Plane configuration model, it can be seen in the schematic of Figure 2, which is a representation of Figure 1.b., but viewed from the side.

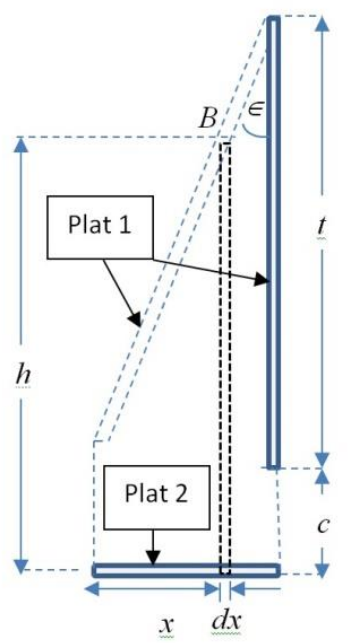

Figure 2. The shape of the line-plane configuration is adopted from Figure 1.b., arranged from two thin plates with plate position 1 forming an angle of inclination of $\in$ to the vertical axis, and it is located above plate 2. The length and width of plate 1 are respectively $s$ and $t$, while the area size of plate 2 is quite large

Suppose plate 1 is in a position above plate 2 and forms a slope angle of $\epsilon<<1$ concerning the vertical axis. The width of plate 1 is equal to $s . t$ and the distance between the two plates when plate 1 is perpendicular to plate 2 is $c$. Because the viewpoint is viewed from the side of the electrode plate 1, then the long trajectory of $s$ is not visible in Figure 2. The capacitance element value in the area element $s d x$ which is located around point $B$ (entered into the image plane) is defined as

$d C=\varepsilon_{0} \frac{d A}{h}=\frac{\varepsilon_{0} s d x}{(x \cot \in+c)}$.

The total capacitance value between the two plates of the line-plane configuration with the position of plate 1 perpendicular to plate 2 can be written as

$C_{\perp}=\lim _{\epsilon \rightarrow 0} \varepsilon_{0} s \int_{x=0}^{t \sin \in} \frac{d x}{[x \cot \in+c]}=\varepsilon_{0} s \ln \left|\frac{t}{c}+1\right|$. 
Using formulation (4), we can calculate the value of the capacitance element of the electrode model with the PSC-P configuration as illustrated in Figure 3.

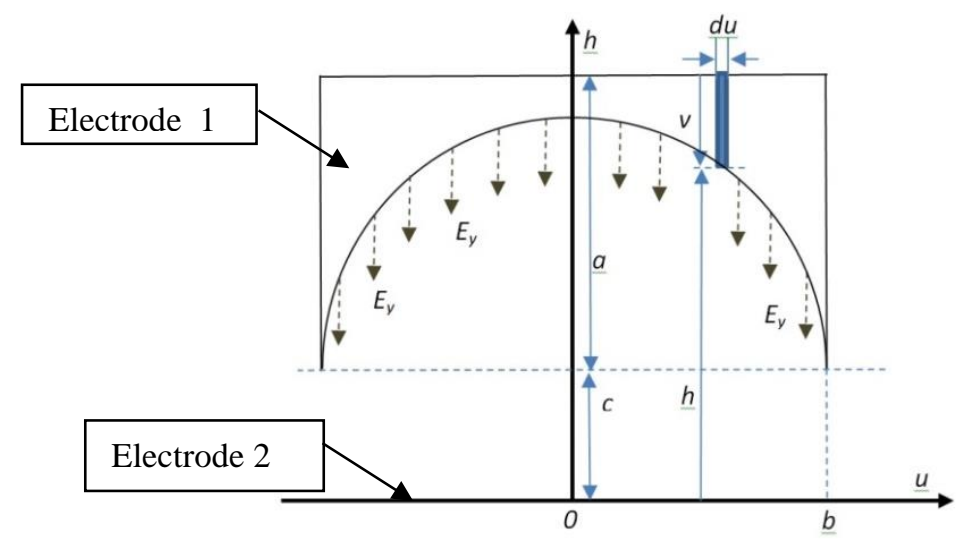

Figure 3. Calculation of the capacitance element from the electrode model with the PSC-P configuration

By comparing the formulation (4) for the capacitance value of the line-plane configuration model with the plate area element as thick as $d u$ in Figure 3, the magnitude of the capacitance element in Figure 3 is

$$
d C_{\perp}=\varepsilon_{0} d u \ln \left|\frac{v}{h}+1\right| .
$$

Also, it is a semicircular form that will connect the curvature of the coordinates $u$ and $h$ to the plate area elements as thick as $d u$,

$u^{2}+(h-c)^{2}=b^{2}, \quad b<c$.

There is a good condition when the electrode model with the PSC-P configuration begins to produce a corona plasma discharge that looks quite clear when it meets the distance $b<c$, as expressed in the requirements of Equation (6). From Equation (6) we can calculate the element value of $d u$ with the boundary conditions range of $u$ coordinate determined as,

$d u=-\frac{(h-c)}{\sqrt{b^{2}-(h-c)^{2}}} d h \quad$ and $\quad 0<u<b$.

where $u$ boundary conditions in Equation (7) only apply to half portion of the plate located on the right of coordinate point 0 in Figure 3, meaning that Equation (5) only applies to calculating half the value of the capacitance located on the right of coordinate point 0 in Figure 3. Based on the symmetrical nature of the plate shape on the left and right of the coordinate point 0 , the total capacitance value can be calculated from Equation (5) by multiplying factor 2.

In Figure 3, there will be a relationship between variables $v$ and $h$ with a constant magnitude of $a+c$ through the equation,

$h+v=a+c$.

Furthermore, through the substitution of Equations (7) and (8) in Equation (5), the capacitance value of the electrode model with the PSC-P configuration will be obtained as 
$C_{\perp}=\varepsilon_{0}\left\{\frac{b}{2} \ln |a+c|-b \ln |c|+b-\left(\frac{\pi}{2}\right) c+\left(\frac{\pi}{2}\right) \sqrt{c^{2}-b^{2}}-\sqrt{c^{2}-b^{2}} \sin ^{-1}\left(\frac{b}{c}\right)\right\}$.

(9)

Equation (9) is the $1 / 2$ part capacitance value for an ordinary electronic circuit case. To calculate the capacitance value originating from the corona plasma discharge case, Equation (9) needs to be modified based on the definition that the corona plasma discharge which produces corona electric current, almost mostly emerges from the surface of the sharp electrode. The solution to the problem of plasma discharge is to add the $k$-shape sharpness factor to the capacitance calculation of the sharp geometry form in Figure 3.

The total capacitance value of the electrode model with the PSC-P configuration for the corona plasma discharge case by adding symmetrical properties of the electrode shape (multiplied by factor 2) and the $k$ shape sharpness factor in Equation (9) is as,

$$
C_{\text {tot }}=\left(\lim _{k \rightarrow \text { big value }} k\right) \mathcal{E}\left\{b \ln |a+c|-2 b \ln |c|+2 b-2 \sqrt{c^{2}-b^{2}} \sin ^{-1}\left(\frac{b}{c}\right)\right\}+\pi \varepsilon_{0}\left(\sqrt{c^{2}-b^{2}}-c\right) \text {. }
$$

Equation (10) is the capacitance value of the corona plasma discharge with the PSC-P electrode configuration for the distance case of $b<c$, wherein the equation, we add the $k$-shape sharpness factor is multiplied to the sharp plate surface form (on the boundary conditions at $u=b$ and $-b$ ). In Equation (10) also, the amount of vacuum space permittivity $\varepsilon_{0}$ has been replaced by the notation $\varepsilon$ for conditions in the air at STP, $900 \mathrm{kHz}$ with a value of $\varepsilon=1.00058986 \varepsilon_{0}$. The value of $\varepsilon_{0}$ is $8.854 \times 10^{-12} \mathrm{~F} \cdot \mathrm{m}^{-1}$.

To calculate the value of the electric current from the capacitance function (10), it is first necessary to calculate the charge and electric field values from the corona plasma discharge event (with $q=(\Delta V)$. $C_{\mathrm{tot}}$ ). If between the two electrodes given a potential difference of $\Delta V$ in Figure 3 ( $\Delta V=V-V_{\mathrm{i}}$, where $V$ is the voltage used and $V_{\mathrm{i}}$ is the corona threshold voltage), then the electric current will flow from electrode 1 to electrode 2, with the largest plasma current flow will come out from the form of the sharp electrode geometry. The value of the electric field according to Gauss's law will be obtained as,

$$
\left(E_{y}\right)_{\perp}=\frac{\Delta V C_{t o t}}{\varepsilon\left(2 b \delta+2 \delta^{2}\right)}
$$

with the area of Gauss defined as $\Delta A=2 b \delta+2 \delta^{2}$ and $\delta=0,1 \mathrm{~mm}$ is the electrode 1 thickness value, with the values of section $2 b \delta$ and $2 \delta^{2}$ are each derived from the form of a half-circle curvature and two sharp electrode ends forms. The total value of the electric current coming out of the electrode system with the PSC-P configuration for the corona plasma discharge case through the concept of electrode geometric calculations as,

$$
I=-\frac{d Q}{d t}=-\frac{\mu}{\Delta V}\left(q E^{2}\right)_{\text {total }}=-\frac{\mu}{\varepsilon^{2}}\left|\frac{\left\{C_{t o t}\right\}^{3}}{\left(2 b \delta+2 \delta^{2}\right)^{2}}\right|\left(V-V_{i}\right)^{2},
$$

where $\mu$ is the permeability value in the air. For comparison with the vacuum condition of $\mu_{0}$, we find $\mu=$ $1.00000037 \mu_{0}$ where $\mu_{0}=4 \pi \times 10^{-7} \mathrm{H} \cdot \mathrm{m}^{-1}$.

Equation (12) is the value of the electric current function as a voltage function of the corona plasma discharge electrode model with a PSC-P configuration. Equation (12) is also a form of Equation (1), where the value of the constant $C_{1}$ is the calculation of the electrode capacitance function. 


\subsection{Experiment Technique}

The reactor equipment schemes used in corona plasma discharge research with PSC-P electrode configuration include:

1. A high-voltage DC generator (4 kV voltage and $25 \mathrm{kHz}$ frequency) connected to the Electrodes with the PSC-P configuration,

2. An electric current measuring device is an analog Multimeter (Ampere meter) with a SANWA brand (type YX-360TREB, voltage $220 \mathrm{~V}$, and frequency $50 / 60 \mathrm{~Hz}$ ). The device is arranged in series with the main circuit,

3. A potential difference measuring device is a digital Multimeter (Voltmeter) with the SANWA brand (type CD771),

4. A device that can convert the $\mathrm{kV}$ to the Volt unit is the $\mathrm{HV}$ probe (Maximum Voltage $40 \mathrm{kV} \mathrm{DC}$, model number: AC $28 \mathrm{kV}$ PD-28, serial number: 01605733) where the electric current is first passed through the HV probe before passing through the digital multimeter (Voltmeter).

Figures 4 (a) and 4 (b) respectively show the equipment circuit schematic and reactor equipment photo for corona plasma discharge experiments with PSC-P electrode configuration.

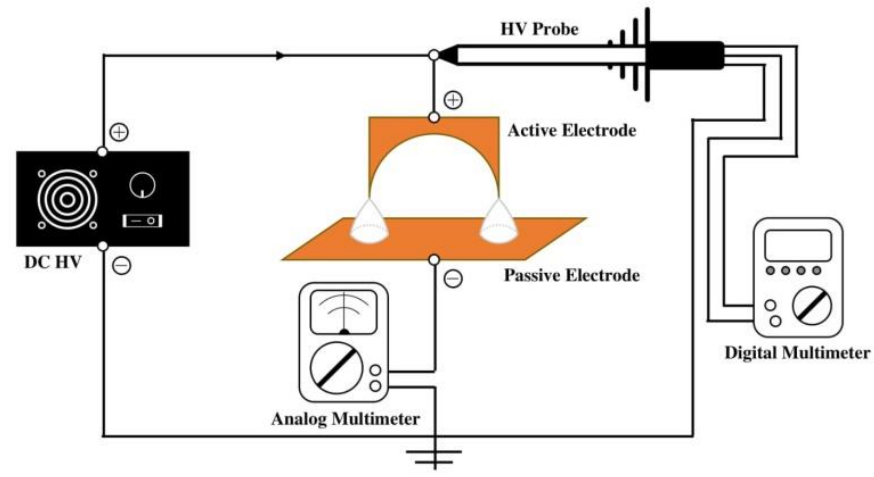

(a)

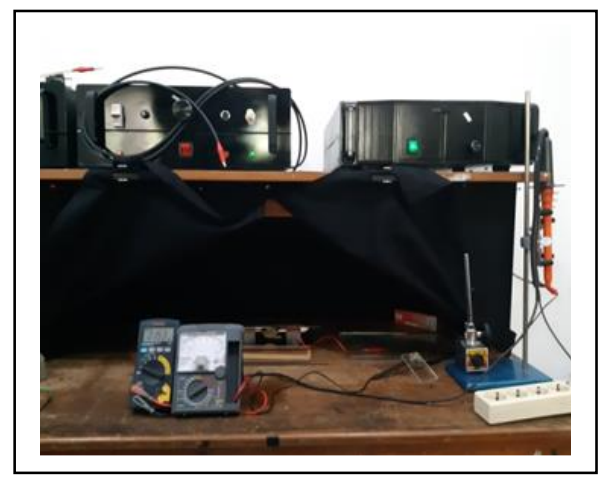

(b)

Figure 4. Research equipment from corona plasma discharge experiments with PSC-P electrode configuration. (a) Equipment circuit scheme. (b) Equipment photos

\section{RESULTS}

The formulation of the electric current in Equation (12) is related to the geometrical concept of the electrode shape with the PSC-P configuration. To prove that the formulation (12) is following the experimental results, it is necessary to be tested through a comparison of the $(I-V)$ characteristics of the simulation results and experimental results by using a size variety of electrodes 1 . This research uses three size variations of the electrodes 1 through the dimensions of the following,

1. Small plate: plate length $a=1 \mathrm{~cm}=0.01 \mathrm{~m}$, plate radius $b=0.5 \mathrm{~cm}=0.005 \mathrm{~m}$,

2. Medium plate: $a=1.5 \mathrm{~cm}=0.015 \mathrm{~m}, b=1 \mathrm{~cm}=0.01 \mathrm{~m}$,

3. Large plate: $a=2.5 \mathrm{~cm}=0.025 \mathrm{~m}, b=2 \mathrm{~cm}=0.02 \mathrm{~m}$.

The results of the corona plasma discharge experiment show almost the same event for the three different sizes electrode case, namely when a corona discharge occurs, bring up the plasmas flow from the lower surface of electrode 1 (anode) to electrode 2 (cathode), with the largest flow emerging from the tip of the lower surface which is the sharpest area of electrode 1 Figure 5 (a), while the experimental photos are shown in Figures 5 (b) -5 (d) for the three-electrode cases. 


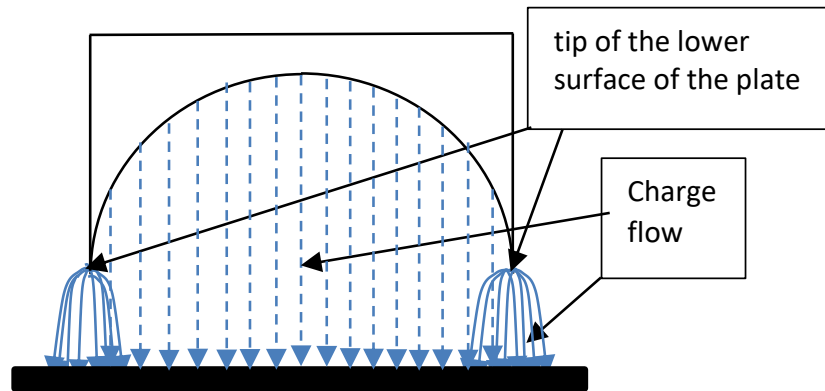

$(a)$.

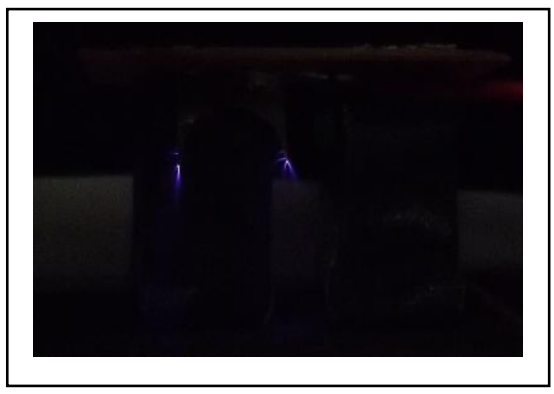

(c)

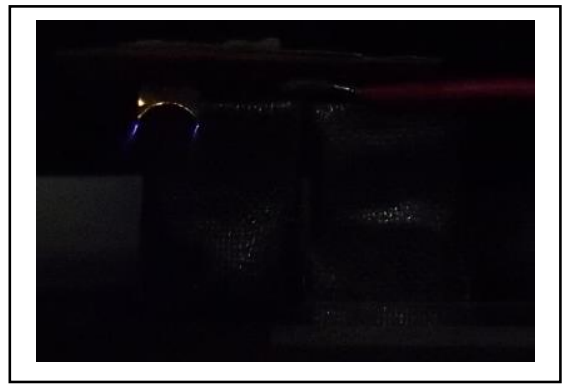

(b)

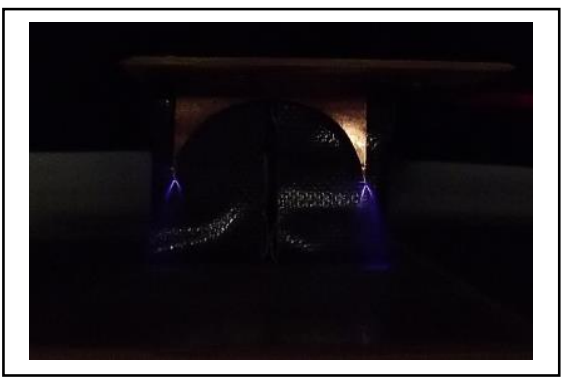

(d)

Figure 5. (a). The plasmas flow of the positive dc corona discharge event in the air that comes out of the electrode model with the PSC-P configuration, with the largest flow coming from the bottom surface of electrode 1 to electrode 2. Figure $(b),(c)$, and (d) are photographs of research results by the concept of plasmas flow for the three different electrode sizes

The (I-V) characteristics curves resulting from the comparison of experimental data (data points) with the numerical calculations (curve lines form) for the three electrode-sizes are illustrated in Figure 6.

For the calculation of the level of accuracy between the numerical simulation curve and experimental data of the $(I-V)$ characteristics at a certain $k$ value, it can be seen in Table 1. The calculation of curves characteristic in Figure 6 along with the level of accuracy uses Python Graphical User Interface (GUI) Programming.

Table 1. Calculation of the level of accuracy between the numerical simulation curve and the point set of experimental data by using the Python GUI Programming for $(I-V)$ characteristics in Figure 6

\begin{tabular}{|l|l|l|l|l|l|l|}
\hline No & Electrode Size & $k$ & $t$-test & $S D$ & $\begin{array}{l}\text { Number of } \\
\text { tangent points }\end{array}$ & $\begin{array}{l}\text { Percentage of } \\
\text { tangent points }\end{array}$ \\
\hline 1. & Small & 1390 & 0.027 & 1.9786 & 7 & $50.00 \%$ \\
\hline 2. & Small & 1335 & 0.0279 & 2.5665 & 8 & $47.06 \%$ \\
\hline 3. & Medium & 1076 & 0.0082 & 1.2171 & 15 & $88.24 \%$ \\
\hline 4. & Medium & 1102 & 0.0741 & 0.5113 & 11 & $68.75 \%$ \\
\hline 5. & Large & 910 & 0.0215 & 18.2943 & 17 & $89.47 \%$ \\
\hline 6. & Large & 959 & 0.0358 & 14.4494 & 12 & $80.00 \%$ \\
\hline
\end{tabular}

In Table 1, we present some measurement accuracy parameters between the numeric simulation curve and experimental data on a certain input value of $k$ which is presented in Figure 6. The measurement parameters include:

- The $t$-test value is a parameter that shows the degree of conformity of the $(I-V)$ curve between the numerical simulation and research data [17]. The smaller the $t$-test value (must be a value less than $0.05)$, the level of suitability of the two functions shows high accuracy. 


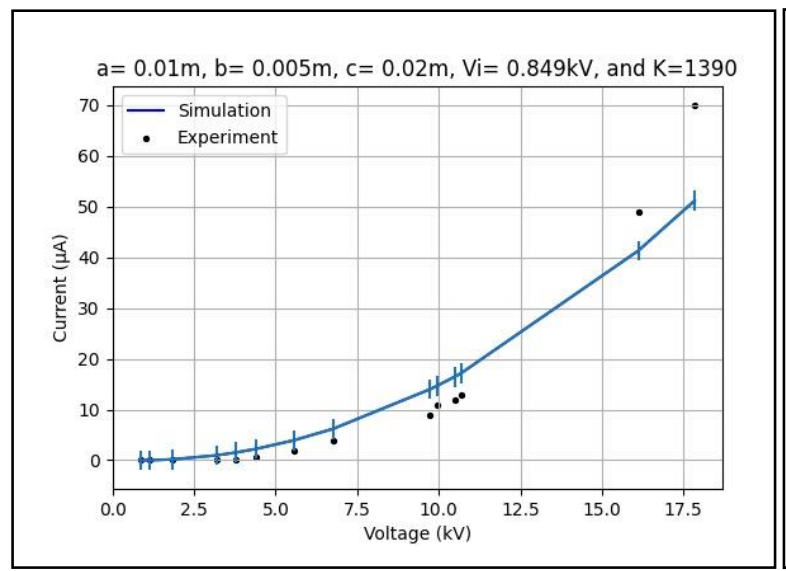

(a)

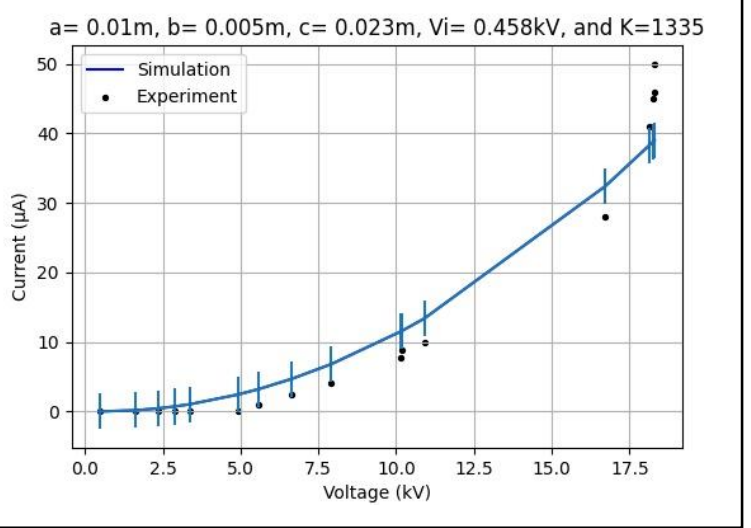

(b)

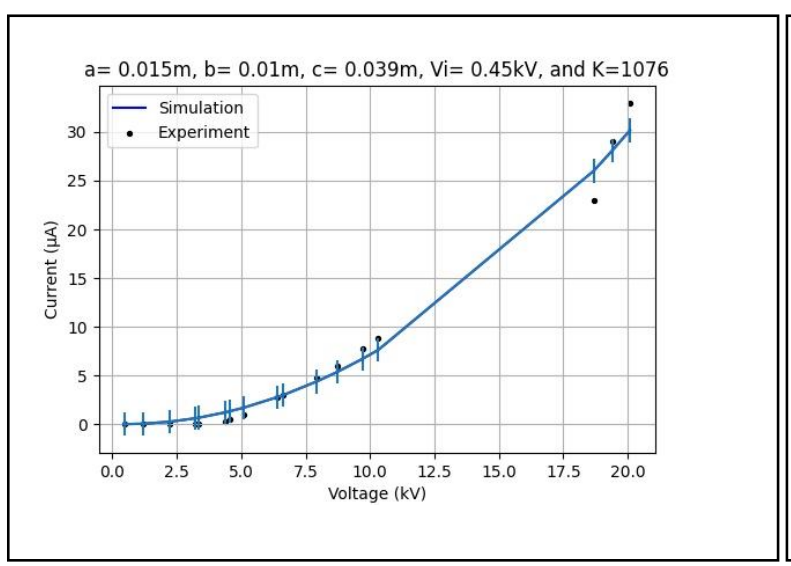

(c)

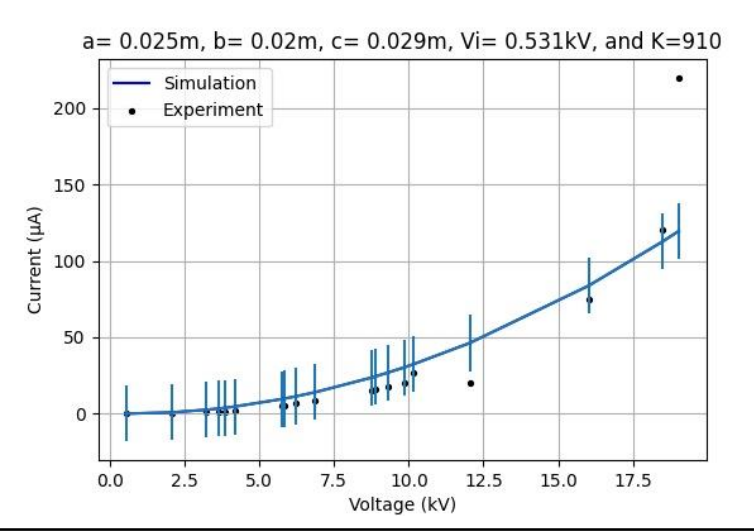

(e)

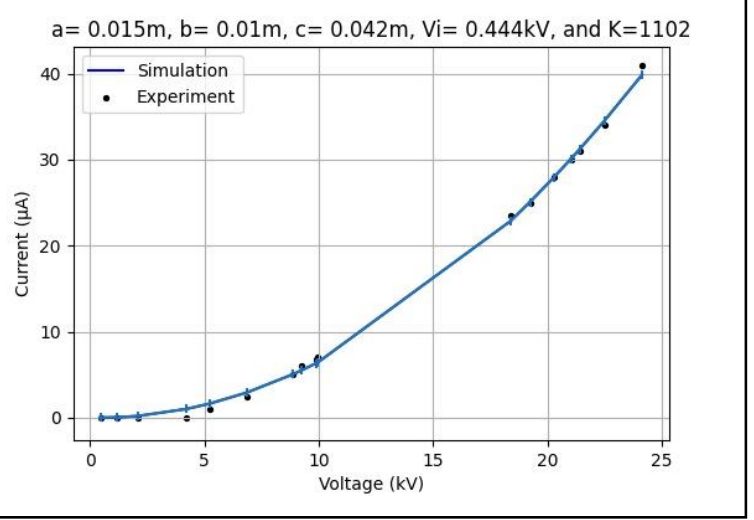

(d)

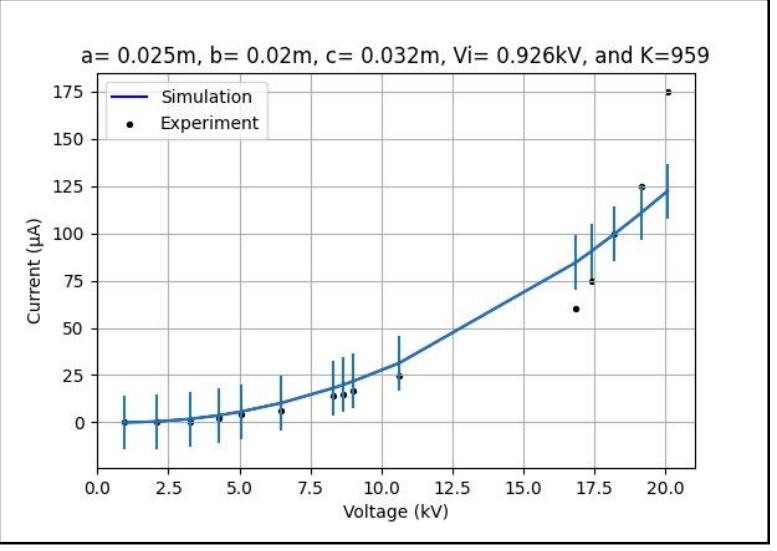

$(f)$

Figure 6. Curves Characteristics of electric current vs voltage (I-V) of the corona plasma discharge with a PSC-P electrode configuration through variations in the value of $c$ for the case $c>b$ of 3 different sizes of the electrode. For Figure $(a)$ and $(b)$ coming from a small plate with a value of $k$ are 1390 and 1335 respectively, Figure $(c)$ and $(d)$ coming from a medium plate with the value of $k$ are 1076 and 1102 respectively and Figure $(e)$ and $(f)$ coming from a large plate with a value of $k$ are 910 and 959 respectively 
- The $S D$ (standard deviation) value in the experiment comes from the distribution of the measured data up to the power order of 2 of the polynomial [18]:

$I=a_{1}+a_{2} V+a_{3} V^{2}$

(13)

where $a_{1}, a_{2}$ and $a_{3}$ are computable constants. The $S D$ formula in Equation (13) is compatible with the current formula in Equation (1) so that it can determine the level of compatibility between the numerical simulation formula and research data.

- The number of tangent points is the number of experimental data points that are tangent to the simulation curve through widening $S D$ values. For the position of the research data adjacent to the simulation curve, the $S D$ value widening is quite narrow (the $S D$ value is small), which indicates a high degree of accuracy between the data position and the simulation curve. For the position of the research data which is far enough from the simulation curve, the $S D$ value widening is made large enough so that the position of the data points can tangent the simulation curve. This event makes the level of accuracy of the position between the experimental data and the numerical simulation curve decreases.

- The percentage of tangent points is the ratio of the number of tangent points with the number of overall experiment data points.

When viewed from Table 1, there are several important notes, namely,

- The $k$ factor value gets smaller if the electrode size gets bigger,

- There is a $t$-test value that exceeds the value of 0.05 , which is for the case of a medium-sized electrode with a value of $k=1102$ which has a $t$-test value of 0.0741 ,

- The standard deviation $(S D)$ value is quite small for small and medium electrode sizes, but the $S D$ value is large enough for large electrode sizes,

- The number of tangent points for small electrode sizes is below 10, so the percentage of tangent points is quite small, while for medium and large electrode sizes, the number of tangent points is large enough so that the percentage of tangent points is quite high.

\section{DISCUSSION AND CONCLUSION}

From the comparison of the numerical simulation graph and research data, we get the $k$ value will be greater if the size of the electrode is getting smaller. The incident was because the smaller the electrode size, the higher the sharpness level at the electrode tip. If the tip of the electrode gets sharper, it will produce a greater flow of plasma coming out of the tip of the electrode, because it has a higher potential gradient. The greater the value of the plasma flow at the end of the electrode is indicated by the multiplication factor $k$ which is also getting bigger in this model so that a large electrode will have the smallest $k$ value and the smallest electrode size will have the largest value of $k$.

There is a $t$-test value of 0.0741 (exceeding the 0.05 limit) for the case of medium-sized electrodes with a value of $k=1102$. This event occurs at a position of research data that is very close to the simulation curve (the smallest $S D$ value is in Table 1). The $S D$ value that has very high accuracy can result in the value of the degree of conformity of the curve $(I-V)$ decreasing so that the $t$-test value gets bigger until it passes the highest allowable limit.

The number of tangent points and their percentage is the combined result of the two factors with the first factor being the level of proximity between the data points and the simulation curve and the second factor being the $S D$ width value. For small-size electrodes, it turns out that the position of data points is close to the numerical curve, but there are still many that do not meet the $S D$ width value requirements which are quite small too within the allowable $t$-test limits. As a result, the number of tangent points and the percentage is small. Medium-sized electrodes have the closest distance between the data points and the numerical curve and many meet the $S D$ width requirement that has the smallest value among all electrode 
sizes. As a result, the number of tangent points and the percentage is quite large. Large size electrodes have a considerable distance between the data points and the curve but many meet the $S D$ width requirement that has the largest value of all electrode forms within the allowable limits of the accuracy of the $t$-test. As a result, the number of tangent points and the percentage is still quite large, even though it has the lowest level accuracy of all electrode sizes.

The general conclusion that can be drawn in this research is that when the electric current is low in value towards a bit high value, the corona discharge event still fulfills the properties of the formulation in Equation (12).

Although the formulation (12) still has some disadvantages, especially in the case of the electric current graph approaching the peak current value, the formulation is compatible with the case of plasma discharge in the electric current before it reaches the peak current, with a taking the certain fitting value of $k$. There is a similar case example regarding the calculation of the formulation $(I-V)$ characteristic of the corona discharge, where it only applies to the low current case as expressed in Equation (2).

In this study, there are still several other drawbacks that come from the experiment, namely

- Lack of accuracy of observations and measuring equipment of plasma reactors,

- Energy loss factor from corona plasma generator reactor equipment.

\section{ACKNOWLEDGMENTS}

This work was financially supported by non-tax revenue (PNBP), Diponegoro University, Semarang, Indonesia under contract No. 1983/UN7.5.8/PP/2020.

\section{CONFLICTS OF INTEREST}

No conflict of interest was declared by the authors.

\section{REFERENCES}

[1] Allen, J., "The plasma-sheath boundary: Its history and Langmuir's definition of the sheath edge", Plasma Sources Science and Technology, 18(1): 014004, (2008).

[2] Stambouli, A. B., Benallal, R., Oudini, N., Mesli, S. M., Tadjine, R., "Control of Dual Frequency Capacitively Coupled Plasma via blocking capacitor and phase angle", The European Physical Journal Applied Physics, 80(1): 10802 (2017).

[3] Saikia, P., Bhuyan, H., Escalona, M., Favre, M., Wyndham, E., Maze, J., Schulze, J., "Study of dual radio frequency capacitively coupled plasma: an analytical treatment matched to an experiment”, Plasma Sources Science and Technology, 27(1): 015014, (2018).

[4] van Veldhuizen, E. M., Rutgers, W. R., "Corona Discharges: Fundamental and Diagnostics", Frontiers in low temperature plasma diagnostics IV: papers, Rolduc Conference Centre, The Netherlands, 40-49, (2001).

[5] Dau, V. T., Dinh, T. X., Terebessy, T., Bui, T. T., "Bipolar corona discharge based air flow generation with low net charge", Journal of Electrostatics, A, 244: 146-155, (2016).

[6] Guan, Y., Vaddi, R. S., Aliseda, A., Novosselov, I., "Analytical model of electro-hydrodynamic flow in corona discharge", Physics of Plasmas, 25(8): 083507, (2018). 
[7] Robinson, M., "Convective Heat Transfer at The Surface of A Corona Electrode", International Journal of Heat and Mass Transfer, 13: 263-274, (1970).

[8] Robinson, M., "Movement of air in the electric wind of the corona discharge", Transactions of the American Institute of Electrical Engineers, Part I: Communication and Electronics, 80: 143-150, (1961).

[9] Robinson, M., “A History of the Electric Wind”, American Journal of Physics, 30: 366-372, (1962).

[10] Robinson, M., "Turbulent Gas Flow and Electrostatic Precipitation", Journal of the Air Pollution Control Association, 18(4): 235-239, (1968).

[11] Bush, J.R., Feldman, P.L., Robinson, M., "High Temperature, High Pressure Electrostatic Precipitation", Journal of the Air Pollution Control Association, 29(4): 365-371, (1979).

[12] Townsend, J. S., "The potentials requires to maintain currents between coaxial cylinders", Philosophical Magazine (London), 28: 83-90, (1914).

[13] Dobranszky, J., Bernath, A., Marton, H., "Characterisation of the plasma shape of the TIG welding arc", International Journal of Microstructure and Materials Properties, 3(1): 126-140, (2008).

[14] Ferreira, G. F. L., Oliveira Jr., O. N., Giacometti, J. A., "Point-to-plane corona: Current-voltage characteristics for positive and negative polarity with evidence of an electronic component", Journal of Applied Physics, 59: 3045, (1986).

[15] Zheng, Y., Zhang, B., He, J., "Current-voltage characteristics of dc corona discharges in air between coaxial cylinders", Physics of Plasmas, 22: 023501, (2015).

[16] Robinson, M., "The Corona Threshold for Coaxial Cylinders in Air at High Pressures", IEEE Transactions on Power Apparatus and Systems, Pas-86: 2, (1967).

[17] Goulden, C. H. "Methods of statistical analysis", USA: John Wiley \& Sons, (1949).

[18] Taylor, J. "Introduction to error analysis, the study of uncertainties in physical measurements", second edition, California: University Science Books, (1997). 